\title{
Elevation Matrix Data in the Evaluation of Keratoconus and Normal Corneas
}

Jaime Tejedor (D) - Francisco J. Gutiérrez-Carmona

Received: November 12, 2021 / Accepted: January 5, 2022 / Published online: January 15, 2022

(C) The Author(s) 2022

\begin{abstract}
Introduction: To determine whether elevation matrix data of the anterior corneal surface could be useful for the diagnosis of keratoconus.

Methods: In a cross-sectional study, subjects aged $10-40$ years with keratoconus $(n=74)$ or age-matched controls $(n=36)$ underwent complete ophthalmological examination, including Scheimpflug corneal topography (Pentacam HR). Exclusion criteria comprised previous ocular surgery, other eye disease, or significant corneal scarring. A raw data matrix of distance measurements to the most anterior corneal point was used to compare each subject with the mean normal cornea. A central $6-\mathrm{mm}$ zone $(6.1 \times 6.1 \mathrm{~mm})$ and two inferior eccentric matrices $(0.4 \times 6.1$ and $1.1 \times 1.1 \mathrm{~mm})$ were
\end{abstract}

Supplementary Information The online version contains supplementary material available at https:// doi.org/10.1007/s40123-022-00454-6.

J. Tejedor $(\bowtie)$

Department of Ophthalmology, Hospital Ramón y

Cajal, C Colmenar km 9100, 28034 Madrid, Spain

e-mail: jaime.tejedor@telefonica.net

J. Tejedor

Department of Neuroscience and Human Vision Laboratory, Universidad Autónoma de Madrid,

Madrid, Spain

F. J. Gutiérrez-Carmona

Department of Surgery, Universidad Alfonso X el

Sabio, Madrid, Spain used. Outcome measures were sensitivity, specificity, positive and negative predictive value, likelihood ratio, accuracy, and odds ratio. Results: Sensitivity of central matrix for the diagnosis of keratoconus was low (6.7\%) whereas specificity reached $94.4 \%$. Sensitivity and specificity were respectively $93.2 \%$ and $94 \%$ for the $6.1 \times 0.4 \mathrm{~mm}$ eccentric matrix and $97.2 \%$ and $97.2 \%$ for the $1.1 \times 1.1 \mathrm{~mm}$ eccentric matrix. Positive predictive and negative predictive values were $71.4 \%$ and $33 \%$, respectively, for the central matrix; $97.1 \%$ and $87.1 \%$; $98.6 \%$ and $94.5 \%$, for the two eccentric matrices, respectively. The likelihood ratio of a positive test was $1.1,16.7$, and 35 , respectively. Sensitivity and specificity of the eccentric matrices were significantly better in the diagnosis of subclinical keratoconus (but not definite keratoconus) than other Pentacam indices. Conclusions: Using eccentric elevation matrix data analysis of the cornea is useful in the detection of keratoconus versus normal corneas.

Keywords: Cornea; Elevation data; Keratoconus; Refractive surgery 


\section{Key Summary Points}

Detection of mild forms or early stages of keratoconus is a challenge, of special interest for keratorefractive surgeons before undertaking refractive surgery, and may be overlooked.

Different indices and criteria have been used, with high sensitivity and specificity, for the diagnosis of keratoconus, like superior-inferior corneal power difference, keratoconus prediction index (KPI), keratoconus index (KCI\%), keratoconus percentage index (KISA\%), and Belin Ambrosio deviation display index (BAD_D).

The present study investigates whether elevation data matrix of the anterior corneal surface could be used to establish the diagnosis of keratoconus.

Using eccentric elevation matrix data of the cornea is useful in the detection of keratoconus versus normal corneas.

In conclusion, analysis of eccentric elevation matrix data of the anterior cornea has high sensitivity and specificity for the diagnosis of keratoconus.

\section{INTRODUCTION}

The diagnosis of keratoconus was initially based on clinical, particularly slit-lamp, findings. Advanced keratoconus is easily identified on the basis of these findings. However, detection of mild forms or early stages of keratoconus is frequently challenging, and may be overlooked. Different indices and criteria have been used for this purpose. Amsler described early forms of keratoconus using photokeratoscopy [1]. Klyce developed algorithms derived from the acquired reflection image of Placido disk videokeratoscopy, for the reconstruction of the anterior surface of the cornea [2]. Indices and methods based on corneal topography were later designed, with varied accuracy, for the diagnosis of mild forms of keratoconus [3-7].

The Amsler Krumeich (AK) classification system has been widely used and is based on refraction, central keratometry, presence of scarring, and central corneal thickness $[8,9]$. The Collaborative Longitudinal Evaluation of Keratoconus (CLEK) study used also changes in vision, keratometry, biomicroscopy findings, corneal scarring, and quality of life related to vision [10]. The AK and CLEK classifications did not incorporate corneal topography analysis.

Rotating Scheimpflug camera technology has the ability to measure the anterior and posterior corneal surface, thus providing additional information of interest in the diagnosis of keratoconus [11-15]. The Belin/Ambrosio enhanced ectasia display uses anterior and posterior elevation data, relative to the commonly used best-fit sphere, and pachymetric data to screen for ectatic change. Belin ABCD keratoconus staging is a very useful approach using different parameters of interest to determine stage, including distance visual acuity, thinnest pachymetry, anterior and posterior corneal surface radius of curvature, and scarring $[15,16]$.

The purpose of this study is to test whether elevation data of the anterior corneal surface could be used to establish the diagnosis of keratoconus. In contrast to refractive power, elevation is not based on axis of curvature, but depicts the height of anterior corneal points to represent the shape of the surface; the corneal elevation profile can represent the shape of the corneal surface more accurately compared with Placido disc-based systems [17]. Viewing raw data lacks qualitative patterns that allow the clinician to separate keratoconus from normal corneas. The reference plane (computer-generated reference surface) most commonly used for corneal elevation data is a sphere with a radius that most closely resembles the overall radius of that corneal surface or a toric ellipsoid. In contrast, we evaluated a matrix mathematical method based on elevation data of Pentacam tomography using raw data relative to the most anterior corneal surface point $z=0$ (not to a best-fit sphere or toric ellipsoid) in order to 
detect keratoconus, by comparing the pattern observed in patients diagnosed with keratoconus, both subclinical and definite, with that in age-matched controls.

\section{METHODS}

\section{Patients}

Patients with the diagnosis of keratoconus aged between 10 and 40 years old and age-matched controls were included in the study, between 2015 and 2019. The study was approved by the Institutional Ethics Committee (UAMA13-4E2192) and adhered to the tenets of the Declaration of Helsinki. Informed consent was obtained from participants or their parents/legal guardians, after explanation of the nature and potential consequences of the study. This is a cross-sectional study including patients examined for the first time in a tertiary referral center and diagnosed with keratoconus (definite or subclinical) or considered as normal controls. Patients who had already been diagnosed with keratoconus and received any kind of treatment previously were excluded.

\section{Procedures}

A complete ophthalmological examination was carried out, which comprised visual acuity (ETDRS logMAR chart), refraction, slit-lamp anterior segment biomicroscopy, intraocular pressure, and funduscopy. Rotating Scheimpflug corneal tomography was used (Pentacam HR, Oculus, Germany). Patients wearing contact lenses discontinued their use for 2 weeks before exam. Pentacam measurements were taken using Pentacam software version 6.08r27. After the patient's chin and forehead were correctly positioned, they were asked to blink before opening both eyes to stare at the fixation target. When adequate alignment was obtained, the automatic release scan started to acquire 25 Scheimpflug images captured within $2 \mathrm{~s}$ for each eye. Only good quality images, as evaluated by the device, were considered valid for the study (quality specification OK). Raw data of the

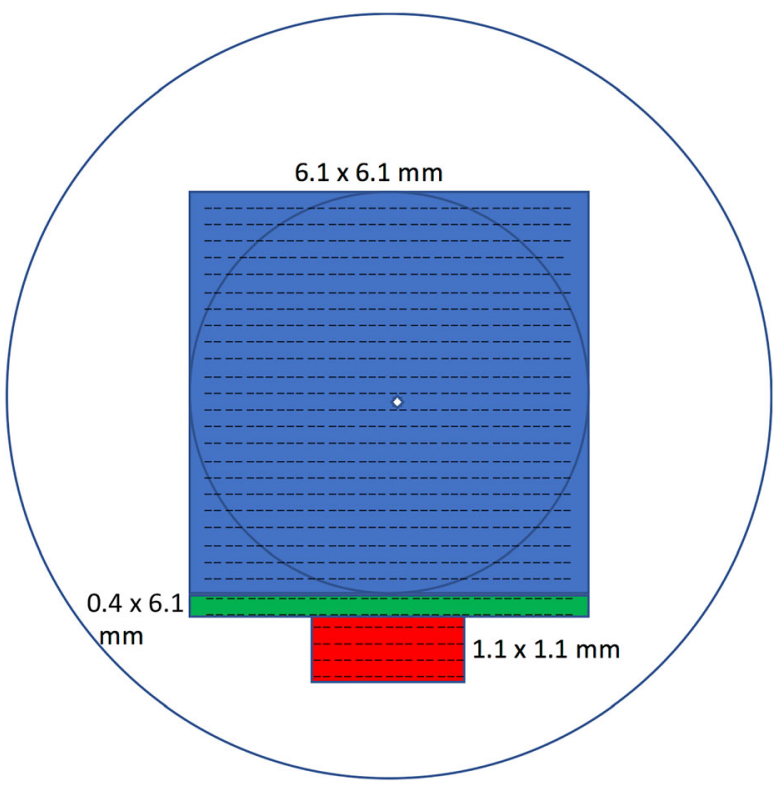

Fig. 1 Representation of the cornea showing how the different elevation matrix data were obtained

distance to the zero corneal point in the $z$ axis of anterior corneal measurements were used in the analysis. We will refer to these as "elevation" data, but they differ from the classical definition of elevation in corneal topography, related to a surface sphere. These data are not displayed in Pentacam elevation maps but obtained from Pentacam software. A matrix of the $6-\mathrm{mm}$ zone $(6.1 \times 6.1 \mathrm{~mm})$ centered on the $(z=0)$ point was initially obtained (Fig. 1) to cover the central to paracentral cornea. Subsequently, rows adjacent but peripheral to this square matrix (superiorly and inferiorly) were analyzed. Data of rows superior to the $6.1 \times 6.1 \mathrm{~mm}$, or more than $0.4 \mathrm{~mm}$ inferior, were not significantly different between controls and patients with keratoconus, so an inferior $0.4 \times 6.1 \mathrm{~mm}$ was chosen for further analysis. The next step was to explore which data showed statistical significance more frequently and consistently in an attempt to reduce the amount of data required for the analysis. The central 1.1-mm data were found to be almost always significantly different, below the $0.4 \mathrm{~mm}$ limit to $1.2 \mathrm{~mm}$, so we also chose a $1.1 \times 1.1 \mathrm{~mm}$ square matrix, inferiorly adjacent to the $6.1 \times 6.1 \mathrm{~mm}$ matrix, for analysis. 


\section{Statistical Analysis}

On the basis of previous pilot data, for a worthwhile difference to detect $75 \mu \mathrm{m}$ between elements in a matrix row, with standard deviation of $80 \mu \mathrm{m}$, at a significance level of 0.01 , to get a power of $90 \%$, at least 34 subjects would be required in each group, (formula used $n_{i}=2 \sigma^{2}$ $\left.\left(\mathrm{Z}_{1-\alpha / 2}+\mathrm{Z}_{1-\beta}\right)^{2} /\left(\mu_{1}-\mu_{2}\right)^{2}\right)$. For statistical analysis we used SPSS (IBM SPSS Statistics Version 22.0. Armonk, NY: IBM Corp) and the Matlab statistical software (The MathWorks Inc, Natick, MA).

The mean matrix of patients with keratoconus was obtained and compared with the mean matrix of control subjects (a mean matrix was the result of adding up the values in each cell and dividing by the number of subjects). The matrix of each keratoconus and control subject was also compared with the mean matrix of control subjects. The Matlab software compares each row of the matrix using Student's $t$ test, with Bonferroni correction of confidence intervals (CIs) for multiple comparisons, so it displays a significance result for each row. The test indicates whether the studied case is within the $95 \% \mathrm{CI}$ limit of controls for that row. The test was considered positive when 95\% of rows yielded a significant test comparison. As a result of the relative naso-temporal enantiomorphic corneal asymmetry, matrix data of left eyes were symmetrically converted to right eye configuration by horizontal flip, so all eyes could be included in the comparisons.

We used the McNemar test to compare sensitivity and specificity, in the diagnosis of keratoconus, of elevation data matrix analysis with those of common indices obtained by Pentacam software.

\section{Classification Criteria}

Our initial analysis included all cases of keratoconus together (definite and subclinical), but subsequently we did separate analyses for definite and subclinical keratoconus subgroups. One eye of each subject was chosen randomly, and subsequently assigned to the subclinical or definite keratoconus subgroup, depending on the characteristics.

The best Pentacam parameter for diagnosing keratoconus is considered to be the Belin/ Ambrosio Deviation Display index (BAD_D) [13]. BAD_D is a comprehensive parameter, which enables a global estimation of the cornea by a combination of elevation and pachymetric data (standard deviation from the average of the normal population for a $\mathrm{D}$ calculated on the basis of a regression analysis that utilizes both anterior and posterior elevation data, and pachymetric data, including thinnest pachymetry, pachymetric distribution, and vertical displacement of the thinnest in relation to the apex).

Criteria used for inclusion with the diagnosis of definite keratoconus were irregular cornea in keratometry, or one biomicroscopic sign of keratoconus (stromal thinning, corneal protrusion at the apex, apical anterior stromal scar, Fleischer ring, Vogt striae, Munson or Rizzuti sign) $[14,16,18-20]$, and $\mathrm{BAD}$ _D greater than 1.45 [21]. Pentacam reports BAD_D values below 1.6 SD as within the normal range (white), those between 1.6 and 2.6 SD as suspicious (yellow), and values of $2.6 \mathrm{SD}$ or above as abnormal (red). However, a value of more than 1.45 is a risk factor for ectasia [21]. Exclusion criteria included previous ocular surgery, eye disease other than keratoconus, or significant corneal scarring.

There is significant disparity in the clinical definition of subclinical keratoconus, forme fruste, and keratoconus suspect in different studies. In the present study, the subclinical keratoconus category included patients with forme fruste keratoconus (FFKC), a condition with high risk of progression to clinical keratoconus, but not keratoconus suspect, defined as an abnormal pattern that resembles keratoconus but with no definitive characteristics $[21,22]$. Forme fruste keratoconus was deemed in a patient's eye with normal clinical biomicroscopic and tomographic appearance and definite keratoconus in the fellow eye. Anterior surface analysis was normal, Pentacam posterior elevation was less than $20 \mu \mathrm{m}$ at the thinnest point $[14,18-21,23]$ (in contrast to early involvement of the posterior corneal surface 
described in keratoconus), and BAD_D was less than 1.45 in all cases $[13,21]$. Although there are arguments indicating that subclinical or forme fruste keratoconus has some initial corneal tomographic or topographic abnormalities, particularly in the posterior corneal surface, we did not apply this criterion. Posterior elevation and back difference elevation on Belin/Ambrosio display had limited sensitivity and specificity to differentiate between forme fruste keratoconus and control eyes [24].

Controls should have normal keratometric and biomicroscopic appearance, no corneal pathology or disease, no previous ocular surgery or irregular corneal pattern, uncorrected visual acuity at least 20/20 (0 logMAR), BAD_D less than 1.45 , spherical equivalent -0.50 to + 0.50 , and refractive cylinder no greater than 0.50 .

\section{RESULTS}

Seventy-five patients met the described keratoconus criteria during the period of study, but one of them was excluded because raw data were not correctly saved. Among them, 39 were assigned to the definite keratoconus subgroup and 35 to the subclinical keratoconus subgroup. Thirty-six controls were identified and recruited during this period. Age at inclusion was not significantly different between keratoconus and control subjects (24.6 years vs 20.16 years, $p=0.4)$, nor was corrected visual acuity (0.2 vs $0 \log$ MAR, $p=0.2$ ). Mean refractive error was myopic in the keratoconus group compared with the control group $(-6.47$ vs +0.21 , $p=0.03)$. Other pachymetric commonly used indices are summarized in Table 1.

The average central square matrix $(6.1 \times 6.1 \mathrm{~mm})$ was not different between controls and patients with keratoconus, except for the inferior rows $(53-61$ rows, $p<0.01)$. The subgroups of definite and subclinical keratoconus also did not differ from controls $(p=0.3$, and $p=0.5$, respectively; except in rows 53-61, $p<0.01$, and rows 55-61, $p<0.01$, respectively). However, average eccentric matrices $(6.1 \times 0.4 \mathrm{~mm}$ and $1.1 \times 1.1 \mathrm{~mm}) \quad$ were

Table 1 Characteristics of patients

\begin{tabular}{lllll}
\hline & KC & KCd & KCs & CG \\
\hline Age (years) & $24.6(22.2-26.9)$ & $30.01(25.6-34.3)$ & $23.7(21.1-26.2)$ & $20.16(18.4-25.3)$ \\
CDVA (logMAR) & $0.2(0.1-0.7)$ & $0.3(0.1-0.7)$ & $0.1(0-0.2)$ & $0(0-0)$ \\
SE (diopters) & $-6.47(-9.82$ to - & $-5.34(-8.93$ to & $+0.45(-0.47$ & $+0.21(-0.52$ \\
& $1.89)$ & $2.18)$ & to +0.61$)$ & to +0.67$)$ \\
ARC & $7.17(5.38-8.80)$ & $7.03(5.40-7.42)$ & $7.65(7.20-8.10)$ & $7.78(7.69-7.88)$ \\
PRC & $5.43(4.95-7.22)$ & $5.64(5.27-6.01)$ & $6.63(6.14-7.12)$ & $6.36(6.23-6.48)$ \\
Thinnest & $479(445-568)$ & $474(450-496)$ & $526(501-568)$ & $557(543-571)$ \\
pachymetry & & & & $0.83(0.30-1.35)$ \\
BAD_D & $6.42(0.40-83.88)$ & $45(3.96-81.58)$ & $0.76(0.44-1.16)$ & $0.15(0.09-0.2)$ \\
IVA & $0.68(0.52-0.94)$ & $0.73(0.53-0.83)$ & $0.14(0.10-0.24)$ & $17.05(15.2-18.9)$ \\
ISV & $65.01(15.75-86.92)$ & $69.05(51.18-78.24)$ & $20.2(15.98-24.41)$ &
\end{tabular}

Mean $(95 \% \mathrm{CI})$

$C D V A$ corrected distance visual acuity, $S E$ spherical equivalent, $A R C$ anterior radius of curvature, $P R C$ posterior radius of curvature, $B A D \_D$ Belin/Ambrosio enhanced ectasia display, $I V A$ index of vertical asymmetry, $I S V$ index of surface variance, $K C$ all keratoconus group, $K C d$ definite keratoconus subgroup, $K C$ s subclinical keratoconus subgroup, $C G$ control group 
different in all rows, in keratoconus vs controls $(p<0.01)$, and in the definite and subclinical subgroups vs controls $(p=0.01, p=0.03$, respectively).

In only 5 of 74 patients with keratoconus, the central square matrix was outside the limits of the $95 \%$ CI of the mean central square control matrix, i.e., a positive test (sensitivity, $6.7 \%$ ). In 34 of 36 control subjects, it was within the $95 \%$ CI limits of the mean central control matrix, a negative test (specificity, 94.4\%) (Supplemental Table 1 in Supplementary Material). Sensitivity was quite similar for the definite and subclinical subgroups $(7.6 \%$ and $5.7 \%$, respectively).

The $6.1 \times 0.4 \mathrm{~mm}$ eccentric matrix was outside the limits of the $95 \% \mathrm{CI}$ of the average control matrix in 69 of 74 patients with keratoconus (sensitivity, 93.2\%); specificity in this group reached 94\% (Supplemental Table 2 in Supplementary Material). Sensitivity in the definite and subclinical subgroups was 37 out of $39(94.8 \%)$ and 32 out of $35(91.4 \%)$, respectively (Supplemental Table 2 in Supplementary Material).

In the $1.1 \times 1.1 \mathrm{~mm}$ eccentric matrix, sensitivity was $97.2 \%(72 / 74)$ for all the patients with keratoconus, but 100\% (39/39) and 97.1\% (34/ 35 ) for the definite and subclinical subgroups, respectively (Supplemental Table 3 in Supplementary Material). Specificity totalled $97.2 \%$.

The positive predictive value (proportion of keratoconus in subjects with positive test) and negative predictive value (proportion of normal controls in subjects with negative test) of each elevation matrix used for the diagnosis of keratoconus are given in Supplemental Tables 1-3 in Supplementary Material.

The likelihood ratio, defined as the likelihood of diagnosing keratoconus in someone with the disease relative to the likelihood of that result in someone without the disease, ranged between 1.02 and 1.38, for the different subgroups, using the central square matrix, and varied between 16.4 and 17.07 for the $6.1 \times 0.4 \mathrm{~mm}$ matrix, and between 34.9 and 36 for the $1.1 \times 1.1 \mathrm{~mm}$ matrix (see Supplemental Table 4 in Supplementary Material).

The odds ratio for each test, defined as the odds of keratoconus when diagnosed relative to the odds of keratoconus when not diagnosed (a cross product in a $2 \times 2$ contingency table), was low in the central matrix analysis (1.03 to 1.4$)$, and ranged between 181.3 and 314.5 using the $6.1 \times 0.4 \mathrm{~mm}$ matrix, and between 1190 and 1365 for the $1.1 \times 1.1 \mathrm{~mm}$ matrix, as shown in Supplemental Table 4 (Supplementary Material).

Using log odds distribution, derived from odds ratio, we obtained statistical significance of this variable, which was not significant in the central square matrix test, but significant in the other two cases (Supplemental Table 4 in Supplementary Material).

Finally, we calculated accuracy as an overall chance or probability of making a correct diagnosis. Accuracy was poor in the central square matrix strategy (35.4-50.7\%), but very good in the two eccentric matrix analyses (92.9-94.6\% and 97.1-98.6\%, respectively) (Supplemental Table 4 in Supplementary Material).

\section{Comparison with Pentacam Indices}

Pentacam analysis provides a number of topometric, pachymetric, and aberrometric indices that are useful in the diagnosis of subclinical and definite keratoconus (Supplemental Table 5 in Supplementary Material). Mean keratometry, BAD_D, and third-order vertical coma were found to be particularly useful in the detection of definite keratoconus vs controls, whereas IVA, ISV, BAD_D, and fifth-order vertical aberration were particularly helpful to detect cases of subclinical keratoconus vs controls [14]. Keratoconus percentage index (KISA\%) was also included in the analysis. Sensitivity and specificity were not significantly different, in general, from the reported indices, for the diagnosis of definite keratoconus, but were better in the diagnosis of subclinical keratoconus, particularly the $1.1 \times 1.1 \mathrm{~mm}$ matrix (Supplemental Table 5 in Supplementary Material).

Figures 2 and 3 (and corresponding Supplemental Figs. 1 and 2, in Supplementary Material, respectively) show two examples of subclinical keratoconus, as diagnosed by the anterior elevation data matrix of $0.4 \times 6.1 \mathrm{~mm}$ and $1.1 \times 1.1 \mathrm{~mm}$, but with the reported 


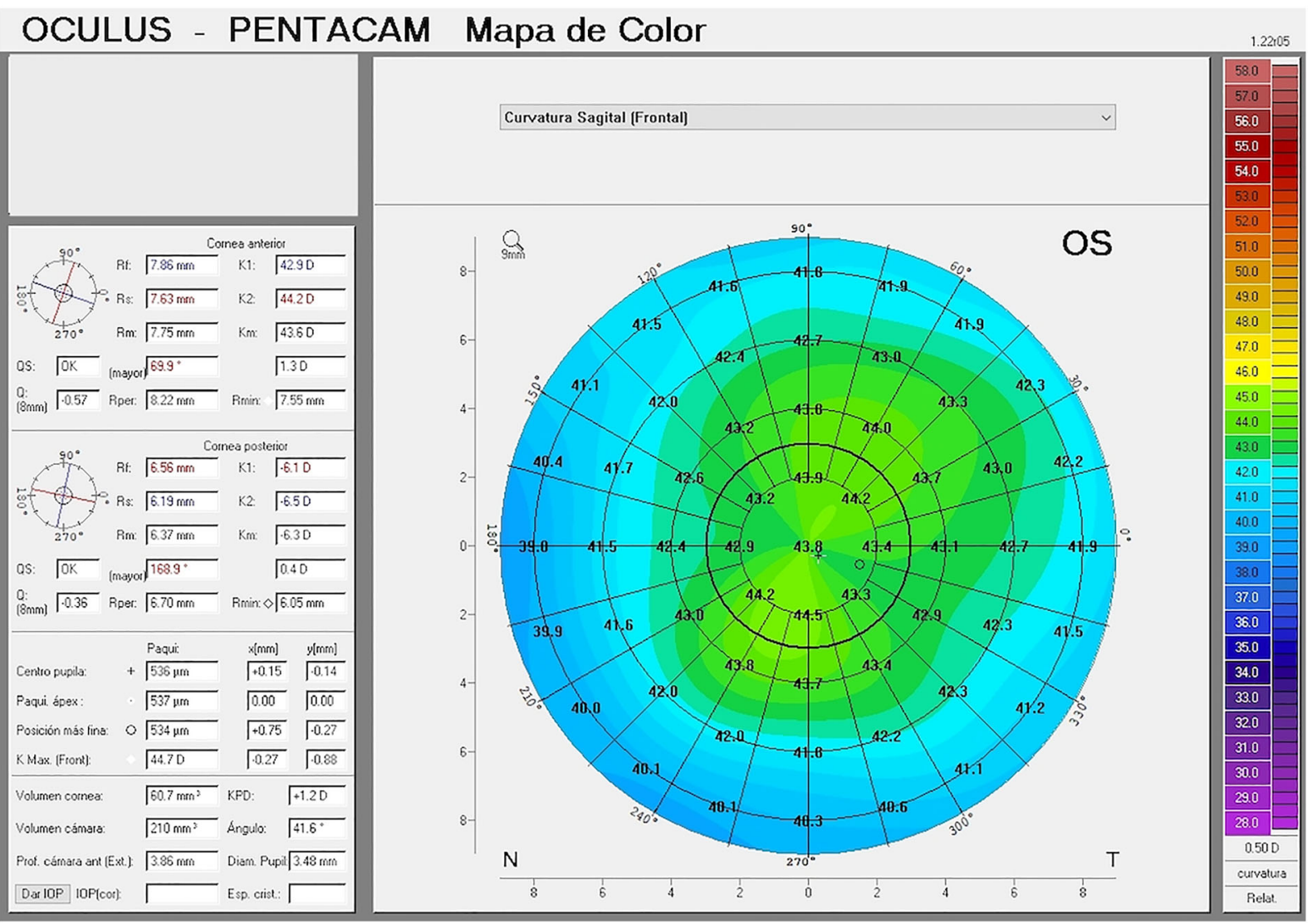

Fig. 2 Corneal map of a patient diagnosed with subclinical keratoconus by elevation matrix data, who had only IHD and BAD Df values (standard deviation from the mean, front surface) outside normal limits (all other

indices within normal limits, or at best only a single index above normal value.

\section{DISCUSSION}

The convenience of using elevation data relative to the most anterior corneal point $(z=0)$ for the diagnosis of keratoconus, in a cross-sectional study, was examined in the present study. Stated another way, it examined whether a matrix representation of the cornea, based on elevation data of the anterior corneal surface, could be used in the detection or diagnosis of keratoconus. Using relatively eccentric elevation matrix data of the cornea is useful in the detection of definite and subclinical keratoconus versus normal corneas. In the present indices within normal limits) (see Supplemental Fig. 1 in Supplementary Material)

analysis, superior eccentric matrix data did not yield statistically significant differences between controls and patients with keratoconus, which could be explained by the fact that less than $1 \%$ of keratoconus cases show changes in the superior cornea [25]. Although there are many different indices and parameters used for the diagnosis or detection of keratoconus, BAD_D was considered to be a good option in Pentacam device technology [13], and therefore it was included in our definition of keratoconus cases. BAD_D is a comprehensive parameter, which enables a global estimation of the cornea by a combination of elevation and pachymetric data. The Belin ABCD classification was used to estimate progression of keratoconus, because it incorporates tomographic 


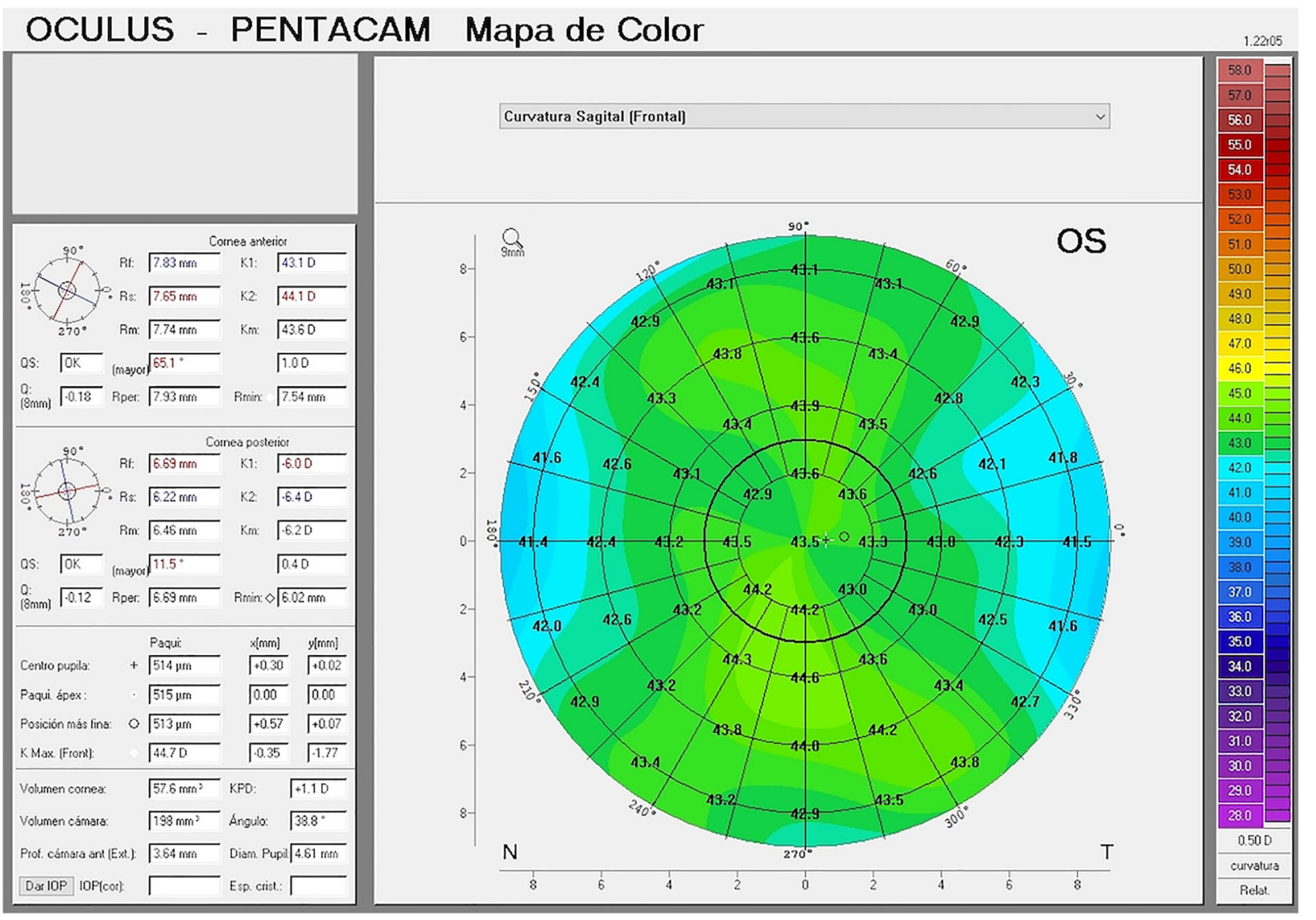

Fig. 3 Corneal map of a patient diagnosed with subclinical keratoconus by elevation matrix data, who had all indices within normal limits (see Supplemental Fig. 2 in Supplementary Material)

data and reflects anatomical and functional changes.

Rabinowitz and McDonnell [6] were the first to describe topographic guidelines for the diagnosis of keratoconus. A maximum $K$ or corneal central power greater than 47.2 , a difference between the two eyes in central $K$ readings of $0.5 \mathrm{D}$ or more, and an inferior-superior corneal power difference $(3 \mathrm{~mm}$ below and above the corneal center) greater than $1.4 \mathrm{D}$ could lead to suspicion of keratoconus (or average of power at 5 points above and 5 points below the center, $1.5 \mathrm{~mm}$ from the center, and $30^{\circ}$ apart). These values had good sensitivity (about 91-96\%) but relatively poorer specificity (81-85\%), and patients diagnosed with keratoconus on the basis of these criteria were followed for years without evidence of definite keratoconus. The keratoconus prediction index (KPI) and keratoconus index (KCI\%) developed by Maeda and Klyce had similar sensitivity (96-98\%) but higher specificity (99\%) [8]. The KISA\% index had sensitivity of $96-99 \%$ and specificity near $100 \%$ [9]. Sensitivity and specificity of the cone location and magnitude index calculated from axial data were $92 \%$ and $100 \%$, respectively [10]. Sensitivity and specificity of using an eccentric elevation matrix pattern compared to the normal population in the diagnosis of keratoconus are higher than in some initially described indices $[6,8]$, but about the same as in other indices described later $[9,10]$. Values are better for the $1.1 \times 1.1 \mathrm{~mm}$ matrix than for the $6.1 \times 0.4 \mathrm{~mm}$ matrix, and better in the definite keratoconus subgroup than subclinical keratoconus subgroup, as expected; however, sensitivity and specificity were very good in the subclinical keratoconus subgroup, especially for the $1.1 \times 1.1 \mathrm{~mm}$ matrix, and as compared with indices reported 
by Pentacam. The latter finding is of particular interest to discriminate between normal and subclinical keratoconus subgroups, for detection of early stages or to identify and exclude subclinical keratoconus cases from refractive surgery.

In general, according to our data, the sensitivity and specificity of eccentric matrices are similar to those of other single indices provided by Pentacam for definite keratoconus, but significantly better for subclinical keratoconus (Supplemental Table 5 in Supplementary Material).

Anterior segment parameters of the eye showed significant alterations with the progression of keratoconus using Pentacam technology [26]. It has been shown that posterior corneal curvature is affected from the early stage of the disorder in keratoconus [27]. Posterior corneal elevation has been described to discriminate very effectively keratoconus from normal corneas, although its efficacy is lower for subclinical keratoconus [24, 28]. We are analyzing elevation matrices of the posterior cornea in the diagnosis of keratoconus, which will be reported in a separate manuscript, to avoid lengthening and profusion of data. Mean posterior corneal elevation was statistically higher in keratoconus and subclinical keratoconus versus normal corneas. Sensitivity and specificity of posterior corneal elevation for the diagnosis of keratoconus were $97.3 \%$ and $96.9 \%$, respectively [28]. Another study found lower values of sensitivity and specificity for posterior elevation to discriminate between forme fruste and controls $(67 \%$ and 59\%, respectively), as well as for back difference elevation on Belin/Ambrosio display to discriminate between forme fruste and controls (74\% and $65 \%$, respectively) [24]. In order to evaluate the adequacy of anterior corneal elevation, we used a different strategy, because we did not use mean elevation, but incorporated data of an elevation matrix, not calculated with respect to a best-fit or surface sphere, but referred to the anterior $(z=0)$ point of the cornea. Sensitivity and specificity were comparable to those obtained using posterior corneal elevation, at least for definite keratoconus [28]. Use of corneal volumetric analysis was also an interesting, recently described, approach for keratoconus detection [29], and yielded sensitivity and specificity (94.8\% and $90.2 \%)$ in the range of or slightly lower than those described in the present investigation.

Currently, the diagnosis of keratoconus is based on clinical suspicion, and further evaluation by biomicroscopy, keratometry, projection- and elevation-based corneal topography, and anterior segment OCT. Cutoff values in corneal indices for the diagnosis of keratoconus, mainly obtained from elevation-based corneal topography like Pentacam, or corneal volumetric analysis, have been suggested, as shown in Supplemental Table 5 (Supplementary Material). Choosing a cutoff value of a single index to conclude the diagnosis of keratoconus is challenging, especially when it is derived from a few parameters for its calculation (BAD_D is a comprehensive parameter obtained by a combination of elevation and pachymetric data). The advantage of using eccentric matrix analysis is that it incorporates many data of corneal surface shape, and has a sensitivity and specificity superior to or equal to that reported for different indices used in the diagnosis of definite keratoconus, but is significantly better in the diagnosis of subclinical keratoconus than the described indices.

Limitations of this study include the moderate sample size, with a satisfactory power of $90 \%$ in the statistical tests employed, and complexity of mathematical analysis. Although the mathematical basis of the methodology described herein is more complex than others (just comparing a single index or parameter), it incorporates more data reflecting the shape of the corneal surface. The described analysis could be incorporated potentially in available slit scan corneal topographers. With expert aid, an algorithm is being created to extract the matrix array of interest from Excel data and compare it with the normal population, so the result is readily available in a few seconds for the clinician, even if not an expert. 


\section{CONCLUSIONS}

Inferior eccentric elevation matrix data may be useful in the diagnosis of keratoconus versus normal corneas (overall accuracy of 92.9-98.6\%). Although there are previously described indices and recent classifications that improve our ability to diagnose keratoconus, the methodology described here is a different strategy that could be incorporated to better the clinician's knowledge of normal and ectatic cornea.

\section{ACKNOWLEDGEMENTS}

We thank the participants of the study.

Funding. No funding or sponsorship was received for this study or publication of this article. The journal's Rapid Service Fee was funded by the authors.

Prior Presentation. Presented in part at the ARVO Annual Meeting 2019, Vancouver, Canada.

Authorship. All named authors meet the International Committee of Medical Journal Editors (ICMJE) criteria for authorship for this article, take responsibility for the integrity of the work as a whole, and have given their approval for this version to be published.

Author Contributions. Jaime Tejedor contributed to the conception and design of the study; acquisition, analysis, interpretation of data; drafting the manuscript or revising it critically; final approval. Francisco J GutiérrezCarmona contributed to the conception and design of the study; interpretation; drafting the manuscript or revising it critically; final approval.

Compliance with Ethics Guidelines. The study was approved by the Institutional Ethics Committee (UAMA13-4E-2192) and adhered to the tenets of the Declaration of Helsinki. Informed consent was obtained from participants or their parents/legal guardians, after explanation of the nature and potential consequences of the study.

Disclosures. Jaime Tejedor and Francisco J Gutiérrez-Carmona confirm that they have nothing to disclose.

Data Availability. The datasets generated during and/or analyzed during the current study are available from the corresponding author on reasonable request.

Open Access. This article is licensed under a Creative Commons Attribution-NonCommercial 4.0 International License, which permits any non-commercial use, sharing, adaptation, distribution and reproduction in any medium or format, as long as you give appropriate credit to the original author(s) and the source, provide a link to the Creative Commons licence, and indicate if changes were made. The images or other third party material in this article are included in the article's Creative Commons licence, unless indicated otherwise in a credit line to the material. If material is not included in the article's Creative Commons licence and your intended use is not permitted by statutory regulation or exceeds the permitted use, you will need to obtain permission directly from the copyright holder. To view a copy of this licence, visit http://creativecommons.org/licenses/by$\mathrm{nc} / 4.0 /$.

\section{REFERENCES}

1. Amsler M. The 'form fruste' of keratoconus. Wien Klin Wochenschr. 1961;73:842-3.

2. Klyce SD. Computer-assisted corneal topography. High-resolution graphic presentation and analysis of keratoscopy. Invest Ophthalmol Vis Sci. 1984;25: 1426-35.

3. Rabinowitz YS, McDonnell PJ. Computer-assisted corneal topography in keratoconus. Refract Corneal Surg. 1989;5:400-8.

4. Holladay JT. Keratoconus detection using corneal topography. J Refract Surg. 2009;25(10 Suppl): S958-62. 
5. Maeda N, Klyce SD, Smolek MK, Thompson HW. Automated keratoconus screening with corneal topography analysis. Invest Ophthalmol Vis Sci. 1994;35:2749-57.

6. Rabinowitz YS, Rasheed K. KISA\% index: a quantitative videokeratography algorithm embodying minimal topographic criteria for diagnosing keratoconus. J Cataract Refract Surg. 1999;25:1327-35.

7. Mahmoud AM, Roberts CJ, Rembach RG, Twa MD, Herderick EE, McMahon TT. CLMI: the cone location and magnitude index. Cornea. 2008;27:480-7.

8. Amsler M. Keratocone classique et keratocone fruste; arguments unitaires. Ophthalmologica. 1946;111:96-101.

9. Krumeich JH, Daniel J, Knulle A. Live-epikeratophakia for keratoconus. J Cataract Refract Surg. 1998;24:456-63.

10. Mc Mahon TT, Szczotka-Flynn L, Barr JT, et al. CLEK study group: a new method for grading the severity of keratoconus: the Keratoconus Severity Score. Cornea. 2006;25:794-800.

11. Ambrosio R Jr, Belin MW. Imaging of the cornea: topography vs tomography. J Refract Surg. 2010;26: 847-9.

12. Ambrosio R Jr, Caiado AL, Guerra FP, et al. Novel pachymetric parameters based on corneal tomography for diagnosing keratoconus. J Refract Surg. 2011;27:753-8.

13. Faria-Correia F, Ramos I, Lopes B, et al. Topometric and tomographic indices for the diagnosis of keratoconus. Int J Keratoconus Ectatic Corneal Dis. 2012;1:92-9.

14. Hashemi $\mathrm{H}$, et al. Pentacam top indices for diagnosing subclinical and definite keratoconus. J Curr Ophthalmol. 2016;28:21-6.

15. Belin MW, Duncan JK. Keratoconus: the ABCD grading system. Klin Monastbl Augenheilkd. 2016;233:701-7.

16. Belin MW, Meyer JJ, Duncan JK, Gelman R, Borgstrom M, Ambrosio R. Assessing progression of keratoconus and cross-linking efficacy: the Belin ABCD progression display. Int J Keratoconus Ectatic Dis. 2017;6:1-10.

17. Belin MW, Khachikian SS. An introduction to understanding elevation-based topography: how elevation data are displayed-a review. Clin Exp Ophthalmol. 2009;37:14-29.
18. Orucoglu F, Toker E. Comparative analysis of anterior segment parameters in normal and keratoconus eyes generated by scheimpflug tomography. J Ophthalmol. 2015;1155:925414.

19. Villavicencio OF, Gilani F, Henriquez MA, Izquierdo L Jr, Ambrósio RR Jr, Belin MW. Independent population validation of the belin/ ambrósio enhanced ectasia display: implications for keratoconus studies and screening. Int J Keratoconus Ectatic Dis. 2014;3:1-8.

20. Belin MW, Ambrósio R. Scheimpflug imaging for keratoconus and ectatic disease. Indian J Ophthalmol. 2013;61:401-16.

21. Ambrósio R, Faria-Correia F, Ramos I, et al. Enhanced screening for ectasia susceptibility among refractive candidates: the role of corneal tomography and biomechanics. Curr Ophthalmol Rep. 2013;1:28-38.

22. Klyce SD, Karon MD, Smolek MK. Screening patients with the corneal navigator. J Refract Surg. 2005;21(5 Suppl):S617-22.

23. Belin MW, Villavicencio OF, Ambrósio RR Jr. Tomographic parameters for the detection of keratoconus: suggestions for screening and treatment parameters. Eye Contact Lens. 2014;40:326-30.

24. Muftuoglu O, Ayar O, Ozulken K, Ozyol E, Akıncı A. Posterior corneal elevation and back difference corneal elevation in diagnosing forme fruste keratoconus in the fellow eyes of unilateral keratoconus patients. J Cataract Refract Surg. 2013;39:1348-57.

25. Weed KH, McGhee CN, MacEwen CJ. Atypical unilateral superior keratoconus in young males. Cont Lens Anterior Eye. 2005;28:177-9.

26. Emre S, Doganay S, Yologlu S. Evaluation of anterior segment parameters in keratoconic eyes measured with the Pentacam system. J Cataract Refract Surg. 2007;33:1708-12.

27. Tomidokoro A, Oshika T, Amano S. Changes in anterior and posterior corneal curvatures in keratoconus. Ophthalmology. 2000;107:1328-32.

28. De Sanctis U, Loiacono C, Richiardi L. Sensitivity and specificity of posterior corneal elevation measured by Pentacam in discriminating keratoconus/subclinical keratoconus. Ophthalmology. 2008;115:1534-9.

29. Cavas-Martínez F, Bataille L, Fernández-Pacheco DG, Cañavate FJF, Alio JL. Keratoconus detection based on a new corneal volumetric analysis. Sci Rep. 2017;7:15837. https://doi.org/10.1038/ s41598-017-16145-3. 